\title{
Paratesticular Adenomatoid Tumor: Clinical Case Report
}

Negre $\mathrm{AA}^{*}$

Department of Urology, Federal University of Tocantins Foundation, Brazil

*Corresponding author: Adelmo Aires Negre, Professor of Urology at the Coordination of the Medicine Course of the Federal University of Tocantins (Uft), Brazil, Tel: 63981212050; Email: adelmourologista@hotmail.com

\section{Case Report}

Volume 4 Issue 2

Received Date: March 05, 2019

Published Date: March 14, 2019

DOI: 10.23880 /oajun-16000159

\section{Abstract}

Purpose: Report of an unusual case in URO-ONCOLOGY, which imposes on the surgeon a real challenge on how to perform the correct diagnosis and preserve the testicular function.

Methods: Bibliographical revision by means of PubMed and Lilacs and case report; a 23 -year- old male patient sent to the Department of Urology of UFT with the complaint of pain in the scrotal and a lump in the right testicular pouch six months ago.

Result: Magnetic resonance imaging confirmed a nodular image, enhanced by gadolinium, measuring $1.4 \mathrm{~cm}$ in the right lower paratesticular region. Laboratory evaluation showed normal tumor markers and normal Testosterone levels. The patient underwent surgery and the anatomopathological diagnosis (with the use of immunohistochemical markers) was an adenomatoid tumor of the epididymis.

Conclusion: The adenomatoid tumor is a rare benign neoplasm of mesothelial origin. It is commonly paratesticular, mainly in the epididymis. Surgical treatment consists of tumor resection and preservation of the testis and spermatic pathway whenever possible.

Keywords: Adenomatoid Tumor; Epididymis; Paratesticular; Neoplasms

\section{Introduction}

Testicular and paratesticular nodules may be of benign or malignant origin. Paratesticular tumors are rare, the adenomatoid is the most common of benign, and can affect the epididymis, testicles, vaginal tunic and spermatic cord, as well as other organs [1-3]. They are usually detected by the presence of a palpable mass or increase in volume in the testicular pouch [4]. The author presents a rare case of paratesticular adenomatoid tumor, proceeding to a brief review of the literature on this subject.

\section{Materials and Methods}

Case report based on data obtained from medical records, physical, laboratory and imaging studies (USG and MRI) of a patient diagnosed with Adenomatoid Tumor of Epididymis, in addition to histopathological analyzes using immunohistochemical markers. Literature reviews have also substantiated this work.

\section{Case Report}

A previously healthy 23-year-old Caucasian man, student, single and childless, noticed a lump in the right 


\section{Open Access Journal of Urology \& Nephrology}

testicular pouch six months ago. No urinary complaints or inflammatory signs. No fever or weight loss history. At the clinical examination, the patient had normal penis and normal testicles. A pain mass of about $1 \mathrm{~cm}$ was detected, palpable at the inferior pole of the right testicle, in the region of the epididymis. Laboratory tests have shown normal tumor markers (Beta HCG, lactate dehydrogenase (LDH), Alpha-fetoprotein) and normal Testosterone levels. The Ultrasonography revealed an extra-testicular hypoechoic nodule of $1.1 \mathrm{~cm} \times 1.1 \mathrm{~cm}$. Magnetic resonance imaging confirmed a nodular image, enhanced by gadolinium, measuring $1.4 \mathrm{~cm}$ in the right lower paratesticular region, and absence of focal lesions in the testes or relation with the testicular albuginea. The patient underwent surgery and the anatomopathological diagnosis (with the use of immunohistochemical markers) was an adenomatoid tumor of the epididymis. The surgical treatment was the right total epididymectomy with preservation of the testis and spermatic pathway.

\section{Result and Discussion}

Paratesticular tumors account for 5\% of intra-scrotal neoplasms, the majority (75\%) of which are benign. The adenomatoid tumor is the most common; accounting for $30 \%$ of all paratesticular masses and has mesothelial origin [5]. In females adenomatoid tumors are found in uterus, fallopian tubes and ovarian hilus. In the male they occur in the epididymis, spermatic cord, prostate and ejaculatory duct. Mostly they arise within or around the lower or upper pole of the epididymis with equal frequency on both sides 4-5. Immunohistochemically, an adenomatoid tumor is positive for markers, such as CK (AE1/AE3) EMA, Cam5.2, CK 5/6, CK7, calretinin, vimentin, WT1, and HBME-1. Other tumor markers, such as AFP, LDH, CEA, and beta-HCG, when measured, are negative, being substantial for the exclusion of malignancy [6]. The intraoperative biopsy may be inconclusive in order to exclude malignancy due to the impossibility of using the immunohistochemical markers.

Sonography is the primary imaging method used to evaluate the origin of a scrotal mass. In case of a paratesticular or extratesticular masses a spatial relationship of mass to testis maybe not clearly defined by ultrasound. MRI shows the enhancement pattern of scrotal adenomatoid tumors and the effect of the tumor on the signal intensity of the adjacent testicular parenchyma and on the enhancement pattern in the adjacent testicular tunica [8]. Extratesticular mass is slightly hypointense relative to testicular parenchyma on T2-weighted images and usually does not enhance more intensely than the testis on contrast-enhanced images.
In the reported case the approach chosen was the scrotal exploration due to preoperative diagnosis of extratesticular mass confirmed by MRI. The surgical treatment was the right total epididymectomy with preservation of the testis and spermatic pathway. Histological findings confirmed the diagnosis of Adenomatoid Tumor of Epididymis. In cases of intratesticular mass with normal values of tumor markers, malignant pathologies cannot be excluded with safety. The imaging tests in this situation are unable to distinguish the benign nature of the lesion.

Thus, an inguinal approach is recommended, similar to that recommended for malignant tumors of testicle, with insulation of the testis of the operative field before the opening of the tunica vaginalis (Radical orchiectomy with high ligation of the spermatic cord).

This case illustrates how MRI evaluation may provide additional morphologic evidence to allow precise localization of the origin of the mass, may also show contrast-enhancement features that enable further confidence of a benign diagnosis, and may allow conservative management. Given its benign nature, the standard treatment of PARATESTICULAR ADENOMATOID TUMOR consists of tumor excision with preservation of the testis, promoting fertility and maintaining the production of testosterone [7]. The present case serves to recall a preponderance of benign paratesticular tumors, alerting the surgeon for the importance of the testicular preservation decision, whenever feasible (Figure 1-3).

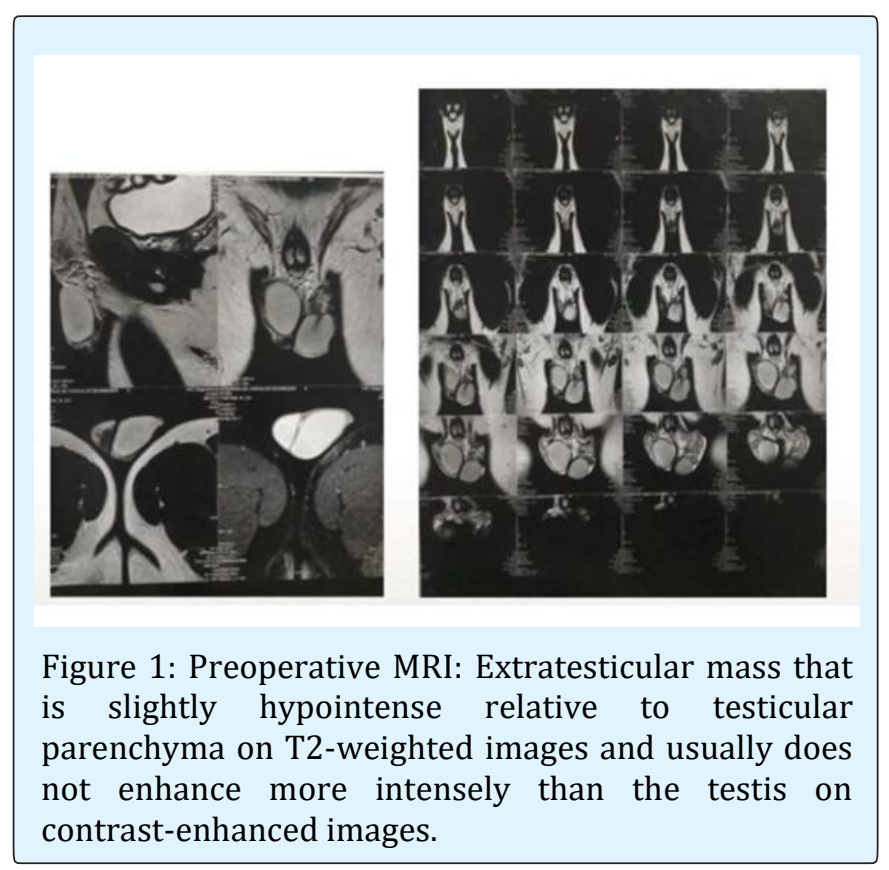




\section{Open Access Journal of Urology \& Nephrology}

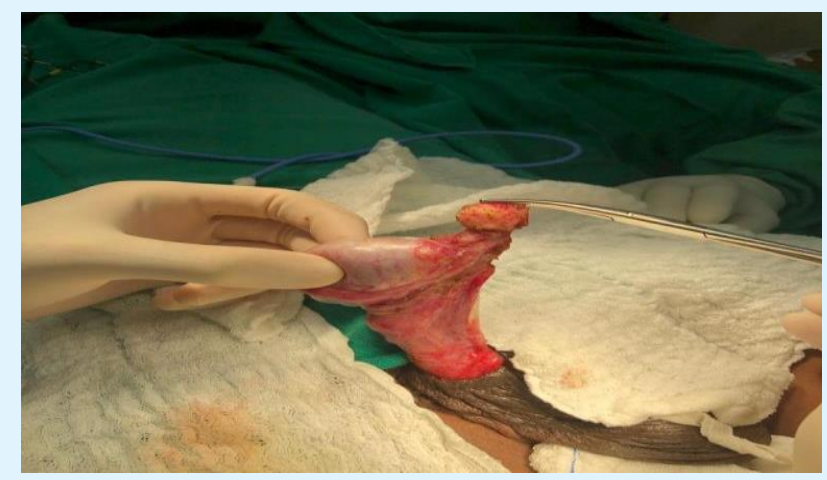

Figure 2: The structure is separate from the testicle.
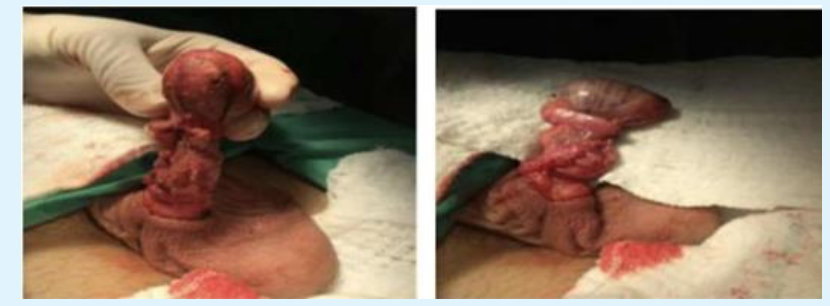

Figure 3: Epididymectomy with preservation of the testis and spermatic pathway.

\section{Ethical Responsibilities}

Protection of people and animals: The author states that the procedures followed are in accordance with the regulations established by the Commission of Clinical and Ethical Inquiry and in agreement with the World Medical Association and the Declaration of Helsinki.

\section{References}

1. Lioe TF, Biggart JD (1993) Tumors of the spermatic cord and paratesticular tissue: a clinicopathological study. Br J Urol 71(5): 600-606.

2. Amin W, Parwani AV (2009) Adenomatoid tumor of the testes. Clin Med Pathol 2:17-21.

3. Liu W, Wu RD, Yu QH (2011) Adenomatoid tumor of the testis in a child. J Pediatr Surg 46(10): E15-E17.

4. Resina Rg, Carranza C, Cordoba JC, Sanchez JMC, Ruiz CC, et al. (2010) Tumor Adenomatoide Paratesticular: Una Serie De Nueve Casos. Actas Urol Esp 34(1): 95100.

5. Luis Sepúlveda, Maria José Freire, David Castelo, Carlos Abrantes, Pedro Moreira, et al. (2015) Tumor adenomatoide paratesticular: a propósito de um caso clínico. Acta Urológica Portuguesa 32(2): 97-100.

6. David J Dabbs (2010) Diagnostic Immunohistochemistry. ( $3^{\text {rd }}$ Edn), WB Saunders, pp: 7-9.

7. Patoulias I, Kaselas C, Patoulias D, Theocharides C, Kalogirou M, et al. (2016) Epididymal Adenomatoid Tumor: A Very Rare Paratesticular Tumor of Childhood. Case Rep Med, Article ID: 9539378.

8. Akbar SA, Sayyed TA, Jafri SZ, Hasteh F, Neill JS (2003) Multimodality Imaging of Paratesticular Neoplasms and Their Rare Mimics. Radiographics 23(6): 1461-1476. 